\title{
Article
}

\section{Some basic properties of Sombor indices}

\author{
Ivan Gutman \\ Faculty of Science, University of Kragujevac, Kragujevac, Serbia.; gutman@kg.ac.rs \\ Communicated by: Muhammad Kamran Jamil \\ Received: 16 December 2020; Accepted: 2 January 2021; Published: 9 January 2021
}

\begin{abstract}
The recently introduced class of vertex-degree-based molecular structure descriptors, called Sombor indices $(\mathrm{SO})$, are examined and a few of their basic properties established. Simple lower and upper bounds for $S O$ are determined. It is shown that any vertex-degree-based descriptor can be viewed as a special case of a Sombor-type index.
\end{abstract}

Keywords: Molecular graph, topological index, degree (of vertex), metric space, degree-space, Sombor index.

MSC: 05C07, 05C09.

\section{Introduction}

$I^{\text {tom }}$

n contemporary mathematical chemistry more than 30 various vertex-degree-based (VDB) molecular structure descriptors (topological indices) have been put forward. Both their physicochemical applicability and mathematical properties were extensively studied; for details see [1-5] and the references cited therein.

The general form of a VDB topological index is

$$
T I=T I(G)=\sum_{i j} F\left(d_{i}, d_{j}\right),
$$

where $d_{i}$ is the degree (= number of first neighbors) of the $i$-the vertex, and the summation goes over all pairs of adjacent vertices of the underlying molecular graph $G . F(x, y)$ is an appropriately selected function with the property $F(x, y)=F(y, x)$.

To each VDB index based on the function $F(x, y)$, it is possible to associate a "reduced" index, replacing $x$ and $y$ by $x-1$ and $y-1$.

In the standard formulation of the theory of VDB topological indices, $\operatorname{TI}(G)$ depends on the vertices of the graph $G$, so that to each vertex a single parameter is associated - namely the vertex degree.

In a recent paper [6], an alternative interpretation of VDB indices has been offered. According to [6], TI(G) is viewed as depending on the edges of the graph $G$, so that to each edge a pair of parameters are associated namely the degrees of the two end-points of the considered edge.

At the first glance, this new interpretation is precisely the same as the traditional one. However, there is a subtle difference.

Let $i j$ denote the edge connecting the $i$-th and the $j$-th vertex of the considered graph $G$. Let $x=d_{i}$ and $y=d_{j}$ be the respective vertex degrees, and assume that $x \geq y$. Then an ordered pair $(x, y)$ represents the edge $i j$.

The pair $(x, y)$ can now be interpreted as a point in a two-dimensional metric space $\mathcal{D}_{2}$ [6]. In [6], Euclidean metric has been employed, as the simplest choice. Then, in particular, the distance of the point $(x, y)$ from the origin is $\sqrt{x^{2}+y^{2}}$. However, it is imaginable to use other distance function [7], but this is left as a task for the future.

The point $(y, x) \in \mathcal{D}_{2}$ is said to be the dual of the point $(x, y) \in \mathcal{D}_{2}$.

In what follows, we refer to $(x, y)$ as to a degree-point of the degree-space $\mathcal{D}_{2}$. Then $(y, x)$ is the dual-degree-point. 
In the later considerations, a VDB topological index will play a distinguished role. This is the first Zagreb index, defined as

$$
Z g=Z g(G)=\sum_{i} d_{i}^{2}
$$

which happens to be historically the first VDB index, conceived as early as in the 1970s [9]. It is long time known [8] that $Z g$ can be rewritten in the form (1) as

$$
Z g=\sum_{i j}\left(d_{i}+d_{j}\right)
$$

The distance $r(x, y)=\sqrt{x^{2}+y^{2}}$ between the degree-point $(x, y)$ and the origin $(0,0)$ is called the degree-radius of the edge $i j[6]$. By summing of $r(x, y)$ over all edges of the underlying graph $G$, and by bearing in mind Equation (1), we arrive at a new VDB structure descriptor, named Sombor index [6]:

$$
S O=S O(G)=\sum_{i j} \sqrt{d_{i}^{2}+d_{j}^{2}}
$$

and its reduced version

$$
S O_{\text {red }}=S O_{\text {red }}(G)=\sum_{i j} \sqrt{\left(d_{i}-1\right)^{2}+\left(d_{j}-1\right)^{2}} .
$$

In the paper [6], several properties of the above defined Sombor indices have been determined. In what follows, we establish a few more.

\section{Simple bounds for $S O$ and $S O_{\text {red }}$}

Theorem 1. Let $\mathrm{Zg}(G)$ be the first Zagreb index of the graph $G$ and let $G$ has m edges. Then

$$
Z g(G)<S O(G) \leq \frac{1}{\sqrt{2}} Z g(G)
$$

and

$$
\mathrm{Zg}(G)-2 m<S O_{\text {red }}(G) \leq \frac{1}{\sqrt{2}}[\mathrm{Zg}(G)-2 m]
$$

Equality on the right-hand side of (5) and (6) holds if and only if the graph $G$ is regular or each of its components is regular.

Proof. Let $a \geq b \geq 1$ be real numbers. Then,

$$
(a+b)^{2}=a^{2}+2 a b+b^{2}>a^{2}+b^{2},
$$

implying

$$
a+b>\sqrt{a^{2}+b^{2}} .
$$

Bearing in mind Equations (2) and (3), we arrive at the left-hand side of (5).

From $(a-b)^{2} \geq 0$, we get

$$
a^{2}+b^{2} \geq 2 a b \Leftrightarrow 2 a^{2}+2 b^{2} \geq a^{2}+b^{2}+2 a b=(a+b)^{2},
$$

implying

$$
\sqrt{2} \sqrt{a^{2}+b^{2}} \geq a+b .
$$

Bearing in mind Equations (2) and (3), we arrive at the right-hand side of (5). Equality holds if $d_{i}=d_{j}$ for all edges of the graph $G$, i.e., if each component of $G$ is a regular graph. If $G$ is connected, then equality holds if and only if $G$ is a regular graph.

The inequalities (6) are obtained by replacing in (7) and (8) $a$ and $b$ by $a-1$ and $b-1$, respectively. 
The bounds (5) and (6) have a far-reaching consequence: The Sombor index and its reduced form behave closely similar to the first Zagreb index $Z g$. A great variety of mathematical properties of $Z g$ have been determined (for details see the survey [10]). Among these are numerous lower and upper bounds. These all could now be applied also to the Sombor and reduced Sombor indices.

In our opinion, the indices $S O$ and $S O_{\text {red }}$ are not of great applicability in QSPR and QSAR studies (since by Theorem 1, they only slightly differ from $Z g$ ). Their true value lies in their new interpretation, offering novel insights thanks to the use of the associated metric space.

\section{Any VDB index is a Sombor-type index}

In the paper [6], it was demonstrated that a particular VDB descriptor, namely the Albertson index is related to the distance between the degree-points and their duals. This means that the Albertson index, introduced a quarter-of-century ago [11], happens to be of Sombor-type. In [6], this concealed property of the Albertson index appeared to be a kind of surprise. It now becomes clear that this was no exception whatsoever.

We now show that any VDB index can be viewed as being of Sombor-type, i.e., as being related to the distance between the degree-points and some other elements of the degree-space.

In order to see this, to any degree-point $(x, y)$ (pertaining to the edges of the corresponding graph $G$ ), we associate another degree point $(x+F, y+F) \in \mathcal{D}_{2}$, where $F$ is the function specified in formula (1). Then the Euclidean distance between these two degree-points is

$$
\sqrt{[(x+F)-x]^{2}+[(y+F)-y]^{2}}=\sqrt{2} F
$$

which by summation over all edges of the graph $G$, and by taking into account Equation (1), becomes equal to $\sqrt{2} T I(G)$.

By the very same argument, we conclude that any reduced VDB index can be viewed as a reduced Sombor-type index.

Conflicts of Interest: "The author declares no conflict of interest."

\section{References}

[1] Dehmer, M., Varmuza, K., \& Bonchev, D. (Eds.) (2012). Statistical Modelling of Molecular Descriptors in QSAR/QSPR. Weinheim: Wiley-Blackwell.

[2] Devillers, J., \& Balaban, A. T. (Eds.) (1999). Topological Indices and Related Descriptors in QSAR and QSPR. Amsterdam: Gordon \& Breach.

[3] Karelson, M. (2000). Molecular Descriptors in QSAR/QSPR. New York: Wiley.

[4] Kulli, V. R. (2020). Graph indices, in: Pal, M., Samanta, S., \& Pal A. (Eds.), Handbook of Research of Advanced Applications of Graph Theory in Modern Society. Hershey: Global, pp. 66-91.

[5] Todeschini, R., \& Consonni, V. (2009). Molecular Descriptors for Chemoinformatics. Wienheim: Wiley-VCH.

[6] Gutman, I. (2021). Geometric approach to degree-based topological indices: Sombor indices. MATCH Communications in Mathematical and in Computer Chemistry, 86, 11-16.

[7] Deza, M. M., \& Deza, E. (2012). Encyclopedia of Distances. Heidelberg: Springer.

[8] Došlić, T., Furtula, B., Graovac, A., Gutman, I., Moradi, S., \& Yarahmadi, Z. (2011). On vertex-degree-based molecular structure descriptors. MATCH Communications in Mathematical and in Computer Chemistry, 66, 613-626

[9] Gutman, I., \& Trinajstić, N. (1972). Graph theory and molecular orbitals. Total $\pi$-electron energy of alternant hydrocarbons. Chemical Physics Letters, 17, 535-538.

[10] Borovićanin, B., Das, K. C., Furtula, B., \& Gutman, I. (2017). Bounds for Zagreb indices. MATCH Communications in Mathematical and in Computer Chemistry, 78, 17-100.

[11] Albertson, M. O. (1997). The irregularity of a graph. Ars Combinatoria, 46, 219-225.

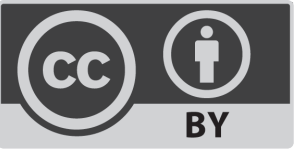

(C) 2021 by the authors; licensee PSRP, Lahore, Pakistan. This article is an open access article distributed under the terms and conditions of the Creative Commons Attribution (CC-BY) license (http://creativecommons.org/licenses/by/4.0/). 